\title{
Laurent Poupard (textes) et Sonia Dourlot (photographies), Autour de la montre en Pays
} horloger : Doubs

Lyon, Lieux Dits, collection « Images et patrimoine », 2019

\section{Sandrine Girardier}

\section{(2) OpenEdition}

Édition électronique

URL : http://journals.openedition.org/artefact/5143

DOI : 10.4000/artefact. 5143

ISSN : 2606-9245

Éditeur :

Association Artefact. Techniques histoire et sciences humaines, Presses universitaires du Midi

Édition imprimée

Date de publication : 15 décembre 2019

Pagination : 360-365

ISBN : 978-2-8107-0667-9

ISSN : 2273-0753

\section{Référence électronique}

Sandrine Girardier, «Laurent Poupard (textes) et Sonia Dourlot (photographies), Autour de la montre en Pays horloger: Doubs", Artefact [En ligne], 11 | 2019, mis en ligne le 27 novembre 2020, consulté le 30 novembre 2020. URL : http://journals.openedition.org/artefact/5143; DOI : https://doi.org/ 10.4000/artefact.5143

Ce document a été généré automatiquement le 30 novembre 2020.

\section{cc) (†) $\ominus$}

Artefact, Techniques, histoire et sciences humaines est mise à disposition selon les termes de la Licence Creative Commons Attribution - Pas d'Utilisation Commerciale - Pas de Modification 4.0 International. 


\section{Laurent Poupard (textes) et Sonia Dourlot (photographies), Autour de la montre en Pays horloger : Doubs}

Lyon, Lieux Dits, collection « Images et patrimoine », 2019

\section{Sandrine Girardier}

\section{RÉFÉRENCE}

Laurent Poupard (textes) et Sonia Dourlot (photographies), Autour de la montre en Pays horloger : Doubs, Lyon, Lieux Dits, collection « Images et patrimoine », 2019, 126 p.

1 L'ouvrage pose le constat suivant: après la Première Guerre mondiale, l'horlogerie française se cantonne à la région de la Franche-Comté, alors qu'elle s'était développée, dès le $\mathrm{XVI}^{\mathrm{e}}$ siècle, à partir de Blois puis de Paris.

2 L'objectif de la publication est explicité à l'emplacement des remerciements. Il s'agit de dresser un état des lieux des sites de production des diverses parties de la montre, réalisées dans les localités comprises dans le Pays horloger, soit la partie qui marque la frontière avec la Suisse dans le département du Doubs. Cet inventaire, résultat d'un important travail de documentation, liste les entreprises horlogères passées et présentes qui maillent le territoire considéré. Il vise notamment à mettre en valeur et à promouvoir l'héritage industriel, mais aussi culturel, technique et économique de la région, à l'aune de la fondation du « Parc naturel régional du Doubs horloger » (p. 2). Il est aussi question d'affirmer la relation avec la partie helvétique de la chaîne du Jura, également concernée par l'industrie horlogère, dans l'optique d'obtenir la reconnaissance des "savoir-faire en mécanique horlogère et mécanique d'art » au titre du Patrimoine immatériel de l'Unesco, dont le dossier de candidature porté par un comité binational franco-suisse a été déposé en 2019. Le projet ambitionne ainsi d'« entretenir la mémoire de cette industrie fondatrice tout en préservant la qualité des 
paysages qu'elle a contribué à façonner » afin de constituer « un axe de travail majeur du futur parc naturel régional » (p. 41).

3 La partie introductive (p. 5-41) pose les bases de l'inventaire à suivre (p. 44-125). Une brève contextualisation du territoire concerné par le projet est suivie d'un rappel des principales évolutions techniques horlogères et des personnalités les plus saillantes associées aux avancées relatives à la mesure du temps.

4 L'implantation horlogère dans le Haut-Doubs se lit à travers le développement d'une industrie rurale, attestée dans la région depuis la fin $d u \mathrm{XvIII}^{\mathrm{e}}$ siècle. On assiste progressivement à une spécialisation dans la fabrication de composants de la montre en fonction des villages. Ébauches, échappements, roues à cylindre, balanciers ou boîtes sont réalisés dans le cadre de l'établissage, mode de production étudié par Philippe Blanchard ${ }^{1}$. Durant le xIX ${ }^{e}$ siècle, des centaines de familles, généralement constituées en entreprises, fabriquent pièces, outils et machines d'établi, qui sont d'abord commercialisés localement. L'arrivée du chemin de fer en 1884 (Besançon-Le Locle via Morteau) facilite le transport des marchandises et des individus tandis que l'électrification permet non seulement de prolonger l'éclairage dans les maisons et les ateliers, mais donne l'élan nécessaire au développement industriel de la région. Un changement de paradigme intervient également avec la construction progressive d'usines à la fin du XIX $x^{e}$ siècle, même si la production au sein d'ateliers éparpillés ne cesse pas pour autant. L'évolution de cette industrie se poursuit pendant le $\mathrm{xx}^{\mathrm{e}}$ siècle, en dépit des crises économiques ou techniques qui se succèdent.

5 La partie principale de l'ouvrage, « Un patrimoine en images », présente nominalement les entreprises liées aux étapes de fabrication des composants de la montre: mouvement (ébauche, échappement, balancier, pierres, décolletage, module à quartz), habillage (boîte, cadran, aiguilles et glace) et traitements des composants (polissage, matage, bleuissage, galvanoplastie, etc.). L'inventaire se poursuit par les lieux de fabrication des outils et des machines (Montécheroux et Les Gras) et par les fournituristes, qui approvisionnent rhabilleurs et fabricants des composants nécessaires. Sont ensuite présentées, par localité, les entreprises qui s'occupent de l'assemblage, et qui concrétisent donc la fabrication de la montre (Morteau, Villers-leLac, Charquemont et Maîche). Un encart est consacré aux écoles horlogères (Besançon et Cluse d'abord, Morteau ensuite) et aux musées d'horlogerie (Morteau et Villers-leLac) où la question de la transmission et de la conservation des savoir-faire est évoquée ${ }^{2}$. L'état des lieux se termine sur des considérations liées à l'architecture industrielle horlogère. Parmi ses principales caractéristiques figure le percement de fenêtres dans les salles hautes des lieux de production, certains élevés ex-novo, mais généralement des anciennes fermes adaptées, dès 1850 , à cette activité, même si la double fonction de production et d'habitation perdure. La manufacture indépendante n'émergera véritablement dans le Haut-Doubs qu'après la Deuxième Guerre mondiale.

6 Les 51 notices d'entreprises sont conçues suivant la même structure : un bref historique comprenant les fondateurs, les spécificités techniques, les étapes (réorganisation, fusion, rachat) et les éventuelles raisons de son effondrement. Les notices sont également complétées d'un ensemble iconographique comportant des visuels représentant le bâtiment, une ébauche de montre, une vue d'atelier et pour certains, un en-tête de lettre de l'entreprise recensée.

7 Essentielle à poser les bases de cet état des lieux, l'introduction ne permet toutefois pas de cerner de manière claire le positionnement de l'ouvrage. Les diverses appellations 
qui y sont convoquées - Pays horloger, Doubs, Jura, Franche-Comté, France voisine, horlogerie jurassienne et ainsi de suite - auraient mérité une contextualisation plus fine, évitant de ce fait d'associer des désignations géographiques ou historiographiques à des propos promotionnels et politiques ${ }^{3}$. Ceci dit, le rabat de la couverture présente une carte de la région, qui aurait gagné à être plus précise, et permet de visualiser la proximité territoriale entre la France et la Suisse, fondamentale pour comprendre le fonctionnement industriel, économique et technique de l'horlogerie aux $\mathrm{XVIII}^{\mathrm{e}}$, $\mathrm{XIX}^{\mathrm{e}}$ et $\mathrm{xx} \mathrm{e}^{\mathrm{e}}$ siècles.

8 Depuis la seconde moitié du xvIII ${ }^{\mathrm{e}}$ siècle en effet, l'horlogerie française est fortement tributaire de l'espace helvétique et de la principauté de Neuchâtel en particulier, qui appartient alors à la Prusse. Dans les Montagnes neuchâteloises, et dans les villages puis les villes de La Chaux-de-Fonds et du Locle, l'horlogerie connaît une forte évolution. L'industrie dite dispersée se pratique ici dans un contexte exempt de contraintes corporatives où les réseaux de distribution sont bien établis et où sont pratiqués, dans un premier temps, des prix hautement concurrentiels. Industrialisation et rationalisation de la production confirment la poussée horlogère de la Suisse au $\mathrm{XIX}^{\mathrm{e}}$ siècle et c'est aussi à travers ce prisme de lecture que l'auteur construit son propos.

9 L'importance et la durabilité des relations entre la France et la Suisse concernent de nombreux aspects de l'industrie horlogère : la réalité transnationale de la production et de la distribution de pièces et d'outils en constitue le noyau. Cette proximité se perçoit à travers la fourniture clandestine de pièces qui passent, "sous les sapins » (p. 13), de part et d'autre de la frontière (France-Suisse-France, Suisse-France-Suisse). La contrebande met ainsi en évidence les stratégies régionales mises en place pour contourner les législations nationales. La formation technique et la pendularité des horlogers français en Suisse sont d'autres éléments qui marquent les liens organiques existant toujours entre les deux pays. S'il ne s'agit pas là de l'objectif premier du travail, il parait néanmoins que la force du propos, distillé tout au long de l'ouvrage, en aurait été décuplée en rassemblant ces considérations de manière thématique ${ }^{4}$.

L'ouvrage s'inscrivant dans la collection « images du patrimoine », il est à regretter que la consultation des illustrations, par ailleurs nombreuses, variées et de bonne qualité, n'ait pas été facilitée par une clé de lecture plus immédiate. On constate en effet que dans la partie introductive, de brèves légendes sont présentées à même les images, tandis que dans la partie dédiée à l'inventaire, ces informations se repèrent (difficilement) à travers la reproduction des silhouettes de leur emplacement sur la page, avec renvoi de lettres (a, b, c...) intégrées dans le corps du texte.

11 Dans l'ensemble, il apparaît qu'une meilleure présentation de l'originalité de l'ouvrage aurait permis de mieux ancrer son propos et son intérêt, car on perçoit parfois difficilement à qui celui-ci est destiné. À des spécialistes? Dans ce cas, s'il n'est pas essentiel de préciser tous les concepts historiographiques questionnés par la communauté de chercheurs intéressés par l'horlogerie ${ }^{5}$, l'apparat bibliographique aurait pu être étoffé et l'ensemble des sources consultées mieux présenté. Au grand public? Auquel cas, les schémas synoptiques présentés au fil de l'ouvrage permettent de mieux comprendre de quels éléments techniques de la montre il est question ici, mais alors les concepts utilisés - paysan horloger, manufacture, industrie, usine, concentration, interchangeabilité de la production, organisation cartellaire et ainsi de suite - auraient pu faire l'objet de renvois bibliographiques et être définis dans un petit glossaire. Aux passionnés de la région? Alors, l'ouvrage dévoile un microcosme 
horloger extraordinairement riche, même si un index des noms des entreprises et des lieux de leur implantation, qui constituent l'épine dorsale de ce travail, en aurait facilité la consultation.

On prend conscience, à la lecture de ce riche inventaire, de la cyclicité des étapes inhérentes aux entreprises du Haut-Doubs, dont le cas d'étude est transposable à d'autres exemples ${ }^{6}$. La circulation des savoirs techniques favorise une première implantation des pratiques artisanales. L'installation des ateliers dans des fermes précède de peu l'électrification de la région à la fin du $\mathrm{xIX}^{\mathrm{e}}$ siècle, ce qui accentue le processus de mécanisation. Par la suite, ces bâtiments font l'objet de constructions annexes, témoignant de la progression industrielle horlogère entre la fin du $\mathrm{XIX}^{\mathrm{e}}$ et le début $\mathrm{du} \mathrm{xx}^{\mathrm{e}}$ siècle. Ces entreprises connaissent des difficultés pendant l'entre-deuxguerres, notamment en lien avec la crise de 1929. Il s'ensuit des remaniements et des fusions. Certaines passent le cap de la crise du quartz des années 1970, d'autres périclitent. Plus récemment, de nombreuses manufactures sont transformées en logements d'habitation, phénomène accentué par le pouvoir d'achat des frontaliers français travaillant en Suisse, fragilisant ce patrimoine horloger et architectural. Face à ce constat, l'ouvrage attire l'attention sur la densité de cette histoire industrielle avant que ses témoins visuels ne disparaissent.

L'ouvrage offre ainsi aux chercheurs une base de données volumineuse permettant de mesurer l'ampleur et la profondeur du tissu industriel, encore vivant dans la région. Il constitue donc un apport bienvenu qui saura susciter davantage d'études spécifiques, comparatives et transfrontalières inhérentes aux entreprises ayant fait l'objet de notices d'inventaire. Celles-ci sont également consultables en ligne ${ }^{7}$ et elles présentent, pour le coup, le détail des sources consultées pour leur établissement. Grâce à la belle complémentarité qui existe entre ces deux supports de contenu, non seulement l'accès en est facilité, mais cet état des lieux pourra évoluer et s'enrichir au fil des travaux, ce qui n'est pas un moindre mérite.

\section{BIBLIOGRAPHIE}

BLANCHARD Philippe, L'établissage. Étude historique d'un système de production horloger en Suisse (1750-1950), Éditions de la Chatière, Chézard-Saint-Martin, 2011.

CARDINAL Catherine (dir.), Apprendre, créer, transmettre : la formation des horlogers, passé et avenir, Institut l'Homme et le Temps, La Chaux-de-Fonds, 1999.

cотTE Michel (dir.), Circulations techniques en amont de l'innovation: hommes, objets et idées en mouvement, Presses universitaires de Franche-Comté/Université de technologie de BelfortMontbéliard, Besançon/Belfort, 2004.

DAUMAS Jean-Claude, GARUFO Francesco, LAMARD Pierre, TISSOT Laurent, Histoires de territoires. Les territoires industriels en question XVIII ${ }^{e}-X^{e}$ siècles, Éditions Alphil, Neuchâtel, 2010. 
GERN Philippe, « Les échanges commerciaux entre la Suisse et la France au XVIII siècle », Journal suisse d'histoire, vol. 21, 1971, p. 64-95.

HILAIRE-PERÉZ Liliane et VERNA Catherine, « La circulation des savoirs techniques du Moyen Âge à l'époque moderne. Nouvelles approches et enjeux méthodologiques », Tracés. Revue de sciences humaines, $\mathrm{n}^{\circ} 16,2009$, p. 25-61.

JUDET Pierre, Horlogeries et horlogers du Faucigny (1849-1934) : les métamorphoses d'une identité sociale et politique, Presses universitaires de Grenoble, Grenoble, 2004.

MARTI Laurence, L'invention de l'horloger, Antipodes \& SHSR, Lausanne, 2003.

MAYAUD Jean-Luc et HENRY Philippe, Horlogeries. Le temps de l'histoire, Neuchâtel-Besançon, 1993-1994, Annales littéraires de l'Université de Besançon, n 591, 1995.

MUNZ Hervé, « La fabrication et les usages politiques du « patrimoine horloger » dans le Pays de Neuchâtel », ethnographiques.org, $n^{\circ}$ 24, juillet 2012, p. 1-20.

\section{NOTES}

1. Blanchard, 2011.

2. cardinal, 1999 ; cotte, 2004 ; Hilaire-Peréz et verna, 2009.

3. Daumas, Garufo, Lamard et Tissot, 2010 ; Munz, 2012.

4. Gern, 1971 ; Mayaud et Henry, 1995.

5. Marti, 2003.

6. Judet, 2004.

7. http://patrimoine.bourgognefranchecomte.fr/connaitre-le-patrimoine/les-ressourcesdocumentaires/acces-aux-dossiers-dinventaire.html

\section{AUTEURS}

\section{SANDRINE GIRARDIER}

Université de Neuchâtel 